CERN-EP/2000-072

18 May 2000

\title{
NEUTRINO PRODUCTION OF OPPOSITE SIGN DIMUONS IN THE NOMAD EXPERIMENT
}

P. Astier ${ }^{1)}$, D. Autiero ${ }^{2)}$, A. Baldisseri ${ }^{3)}$, M. Baldo-Ceolin ${ }^{4)}$, G. Ballocchi ${ }^{2)}$, M. Banner ${ }^{1)}$, G. Bassompierre ${ }^{5)}$, K. Benslama ${ }^{6)}$, N. Besson ${ }^{3)}$, I. Bird ${ }^{2,6)}$, B. Blumenfeld ${ }^{7)}$, F. Bobisut ${ }^{4)}$, J. Bouchez ${ }^{3)}$, S. Boyd $^{8)}$, A. Bueno $^{9,10)}$, S. Bunyatov ${ }^{11)}$, L. Camilleri ${ }^{2)}$, A. Cardini ${ }^{12)}$, P.W. Cattaneo ${ }^{13)}$, V. Cavasinni ${ }^{14)}$, A. Cervera-Villanueva ${ }^{2,15)}$,

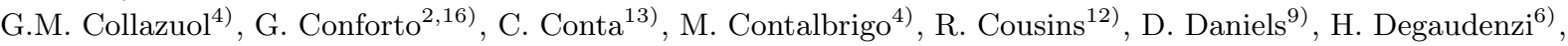
A. De Santo ${ }^{2,14)}$, T. Del Prete ${ }^{14)}$, T. Dignan ${ }^{9)}$, L. Di Lella ${ }^{2)}$, E. do Couto e Silva ${ }^{2)}$, J. Dumarchez ${ }^{1)}$, M. Ellis ${ }^{8)}$, T. Fazio ${ }^{5)}$, G.J. Feldman ${ }^{9)}$, R. Ferrari ${ }^{13)}$, D. Ferrère ${ }^{2)}$, V. Flaminio ${ }^{14)}$, M. Fraternali ${ }^{13)}$, J-M. Gaillard ${ }^{5)}$,

E. Gangler ${ }^{1)}$, A. Geiser ${ }^{2,17)}$, D. Geppert ${ }^{17)}$, D. Gibin ${ }^{4)}$, S. Gninenko ${ }^{18)}$, A. Godley ${ }^{8)}$, J-J. Gomez-Cadenas ${ }^{2,15)}$, J. Gosset ${ }^{3)}$, C. Gößling ${ }^{17)}$, M. Gouanère ${ }^{5)}$, A. Grant ${ }^{2)}$, G. Graziani ${ }^{19)}$, A. Guglielmi ${ }^{4)}$, C. Hagner ${ }^{3)}$, J. Hernando ${ }^{15)}$, D. Hubbard ${ }^{9)}$, P. Hurst ${ }^{9)}$, N. Hyett ${ }^{20)}$, E. Iacopini ${ }^{19)}$, C. Joseph ${ }^{6)}$, F. Juget ${ }^{6)}$, M. Kirsanov ${ }^{16,18)}$, O. Klimov ${ }^{11)}$, J. Kokkonen ${ }^{2)}$, A. Kovzelev ${ }^{13,18)}$, A. Krasnoperov ${ }^{5,11)}$, V. Kuznetsov ${ }^{2,11)}$, S. Lacaprara ${ }^{4)}$, B. Lakic ${ }^{21)}$, A. Lanza ${ }^{13)}$, L. La Rotonda ${ }^{22)}$, M. Laveder ${ }^{4}$, A. Letessier-Selvon ${ }^{1)}$, J-M. Levy ${ }^{1)}$,

L. Linssen ${ }^{2)}$, A. Ljubičić( ${ }^{21)}$, J. Long ${ }^{7)}$, A. Lupi ${ }^{19)}$, E. Manola-Poggioli ${ }^{5)}$, A. Marchionni ${ }^{19)}$, F. Martelli $^{16)}$, X. Méchain ${ }^{3)}$, J-P. Mendiburu ${ }^{5)}$, J-P. Meyer ${ }^{3)}$, M. Mezzetto ${ }^{4)}$, S.R. Mishra ${ }^{9,23)}$, G.F. Moorhead ${ }^{20)}$, L. Mossuz ${ }^{5)}$,

P. Nédélec ${ }^{5)}$, Yu. Nefedov ${ }^{11)}$, C. Nguyen-Mau ${ }^{6)}$, D. Orestano ${ }^{24)}$, F. Pastore ${ }^{24)}$, L.S. Peak ${ }^{8)}$, E. Pennacchio ${ }^{16)}$, H. Pessard ${ }^{5)}$, R. Petti ${ }^{13)}$, A. Placci ${ }^{2)}$, A. Pluquet ${ }^{3)}$, G. Polesello ${ }^{13)}$, D. Pollmann ${ }^{17)}$, A. Polyarush ${ }^{18)}$, B. Popov ${ }^{1,11)}$, C. Poulsen ${ }^{20)}$, P. Rathouit ${ }^{3)}$, C. Roda ${ }^{2,14)}$, A. Rubbia ${ }^{2,10)}$, F. Salvatore ${ }^{13)}$, K. Schahmaneche ${ }^{1)}$, B. Schmidt ${ }^{2,17)}$, T. Schmidt ${ }^{17)}$, M.E. Sevior ${ }^{20)}$, D. Sillou ${ }^{5)}$, F.J.P. Soler ${ }^{2,8)}$ G. Sozzi ${ }^{6)}$, D. Steele ${ }^{6,7)}$, M. Steininger ${ }^{6)}$, U. Stiegler ${ }^{2)}$, M. Stipčević ${ }^{21)}$, T. Stolarczyk ${ }^{3)}$, M. Tareb-Reyes ${ }^{6)}$, G.N. Taylor ${ }^{20)}$,

S. Tereshchenko ${ }^{11)}$, A. Toropin ${ }^{18)}$, A-M. Touchard ${ }^{1)}$, S.N. Tovey ${ }^{2,20)}$, M-T. Tran ${ }^{6)}$, E. Tsesmelis ${ }^{2)}$, J. Ulrichs $^{8)}$,

L. Vacavant ${ }^{6)}$, M. Valdata-Nappi ${ }^{22)}$, V. Valuev ${ }^{11,12)}$, F. Vannucci ${ }^{1)}$, K.E. Varvell ${ }^{8)}$, M. Veltri ${ }^{16)}$, V. Vercesi ${ }^{13)}$, D. Verkindt ${ }^{5)}$, J-M. Vieira ${ }^{6)}$, T. Vinogradova ${ }^{12)}$, M-K. Vo ${ }^{3)}$, F. Weber ${ }^{2,9)}$ T. Weisse ${ }^{17)}$, F. Wilson $^{2)}$, L.J. Winton ${ }^{20)}$, B.D. Yabsley ${ }^{8)}$, H. Zaccone ${ }^{3)}$, K. Zuber ${ }^{17)}$, and P. Zuccon ${ }^{4)}$

\begin{abstract}
The NOMAD Collaboration presents a study of opposite sign dimuon events in the framework of Leading Order QCD. A total of 2714 neutrino- and 115 antineutrino-induced opposite sign dimuon events with $E_{\mu 1}, E_{\mu 2}>4.5 \mathrm{GeV}, 15<E_{\nu}<300 \mathrm{GeV}$ and $Q^{2}>1(\mathrm{GeV} / \mathrm{c})^{2}$ are observed in the Front-Calorimeter of NOMAD during the 1995 and 1996 runs. The analysis yields a value for the charm quark mass of $m_{c}=1.3_{-0.3}^{+0.3}{ }_{-0.3}^{+0.3} \mathrm{GeV} / \mathrm{c}^{2}$ and for the average semileptonic branching ratio of $B_{c}=0.095_{-0.007}^{+0.007}+0.014$. The ratio of the strange to non-strange sea in the nucleon is measured to be $\kappa=0.48_{-0.07-0.12}^{+0.09+0.17}$. The measured rate of charm-induced dimuon relative to single muon, as a function of neutrino energy, is consistent with the slow rescaling hypothesis of heavy quark production.
\end{abstract}


1) LPNHE, Univ. of Paris, Paris VI and VII, France.

2) CERN, Geneva, Switzerland.

3) DAPNIA, CEA Saclay, France.

4) Univ. of Padova and INFN, Padova, Italy.

5) LAPP, Annecy, France.

6) University of Lausanne, Lausanne, Switzerland.

7) Johns Hopkins Univ., Baltimore, MD, USA.

8) Univ. of Sydney, Sydney, Australia.

9) Harvard Univ., Cambridge, MA, USA.

10) ETH Zürich, Zürich, Switzerland.

11) JINR, Dubna, Russia.

12) UCLA, Los Angeles, CA, USA.

13) Univ. of Pavia and INFN, Pavia, Italy.

14) Univ. of Pisa and INFN, Pisa, Italy.

15) IFIC, Valencia, Spain.

16) Univ. of Urbino, Urbino, and INFN Florence, Italy.

17) Dortmund Univ., Dortmund, Germany.

18) Inst. Nucl. Research, INR Moscow, Russia.

19) Univ. of Florence and INFN, Florence, Italy.

20) University of Melbourne, Melbourne, Australia.

21) Rudjer Bošković Institute, Zagreb, Croatia.

22) Univ. of Calabria and INFN, Cosenza, Italy.

23) Univ. of South Carolina, Columbia, SC, USA.

24) Roma-III Univ., Rome, Italy. 


\section{INTRODUCTION}

A charged current muon-neutrino deep inelastic scattering event produces a single muon at the leptonic vertex and changes the flavour of the initial quark at the hadronic vertex. If the initial quark is a strange (down) quark, a charm quark may be produced via a Cabibbo enhanced (suppressed) transition. The charm quark then fragments into a charmed hadron which may decay semileptonically, producing either a second muon or an electron with its electric charge opposite to that of the muon from the leptonic vertex. This type of event is referred to as an opposite sign dilepton event. If the second lepton is a muon the event is usually labelled as a dimuon event. This process is a powerful and clean probe of both the strange component of the nucleon sea and the kinematics of heavy quark production.

The phenomenon of charm production by neutrinos has been investigated by a number of experiments. Dimuons have been studied in counter experiments : CDHS[1], CHARM II[4], CCFR[3] and FMMF[5]; in bubble chamber experiments such as Col-BNL[6], BEBC[7] and E362[8] at Fermilab; and in the Fermilab emulsion experiments, E531[9] and E564[10]. Bubble chamber experiments generally have too few events to study the parameters of charm production with any precision. It is the counter experiments, and the E531 experiment, which have provided much of our knowledge of neutrino charm production.

This paper presents the results of an analysis of opposite sign dimuon events carried out by the NOMAD collaboration. The distributions of various kinematic variables are compared with a theoretical model of dimuon production, constructed within the framework of leading order QCD, to yield a determination of the charm quark mass, $m_{c}$, the strange quark fraction of the nucleon sea, $\kappa$, and the average semileptonic branching ratio, $B_{c}$. The paper is organised as follows : Section 2 outlines the theoretical description of opposite sign dimuon production within the framework of leading order QCD and Section 3 gives a brief description of the neutrino beam. The detector is described in Section 4 along with a description of the simulation programs used in the analysis (Section 4.2), a description of the data selection process and a discussion of the background determination. Section 5 presents details of the analysis and Section 6 contains a discussion and a comparison with the results of other experiments. Finally, Section 7 summarizes the results.

\section{THEORY}

In the Standard Model, an opposite sign dimuon event is produced when a neutrino interacts, via a charged current, with a strange $(s)$ or down $(d)$ quark, producing a charm $(c)$ quark. The charm quark fragments into a charmed hadron (most frequently a $D$ meson) which can then decay semileptonically resulting in a final state containing two oppositely charged muons : the primary muon which comes from the leptonic vertex and the secondary muon which arises from the decay of the charmed hadron.

The large mass of the charm quark, $m_{c}$, gives rise to an energy threshold which has the effect of suppressing the opposite sign dimuon production rate at low neutrino energies. This is effectively described by the slow rescaling model[11] in which the usual scaling variable, $x=Q^{2} / 2 M \nu$, is replaced by the slow-rescaling variable, $\xi=x\left(1+m_{c}^{2} / Q^{2}\right)$. With this replacement, the leading order cross section for opposite sign dimuon production by neutrino scattering on an isoscalar target may be written[5]

$$
\begin{aligned}
\frac{d^{3} \sigma^{\nu}}{d \xi d y d z}= & \frac{G_{F}^{2} M E_{\nu} \xi}{\pi}\left\{\left[u\left(\xi, Q^{2}\right)+d\left(\xi, Q^{2}\right)\right]\left|V_{c d}\right|^{2}\right. \\
& \left.+2 s\left(\xi, Q^{2}\right)\left|V_{c s}\right|^{2}\right\}\left(1-y+\frac{x y}{\xi}\right) D(z) B_{c}
\end{aligned}
$$

where $G_{F}$ is the Fermi constant; $E_{\nu}$ is the energy of the incident neutrino; $M$ is the mass of the nucleon; $Q^{2}$ is the negative four momentum transfer squared; $x$ and $y$ are the Bjorken variables; $z$ is defined to be the ratio of the momentum of the charmed hadron to the maximum momentum that the kinematics will allow that hadron to possess; $V_{c d}$ and $V_{c s}$ are Cabibbo-Kobayashi-Maskawa (CKM) matrix elements[12] and $u\left(\xi, Q^{2}\right), d\left(\xi, Q^{2}\right)$ and $s\left(\xi, Q^{2}\right)$ are the up, down and strange quark structure functions, respectively. The function $D(z)$, discussed below in Section 4.2, describes the fragmentation of the charm quark into a charmed hadron and $B_{c}$ is the average semileptonic branching ratio for charmed hadron decay. The corresponding cross section for incident antineutrinos is obtained by replacing the quark structure functions with the antiquark structure functions in Eq.(1). It should be noted that the slow rescaling hypothesis is an effective - and, as demonstrated by our data, consistent - description of the heavy quark production. A next-to-leading (NLO) order treatment of the data would yield different values for the structure function related parameters [2]. 
The CERN-SPS wide band beam is produced by $450 \mathrm{GeV} / \mathrm{c}$ protons incident on a beryllium target. The secondary pions and kaons pass through a large angle aluminium collimator and are focussed by a system of magnetic lenses, which focus(defocus) positive(negative) mesons. The particles decay in a $290 \mathrm{~m}$ long evacuated decay tunnel and the decay products then pass through an earth and iron shield which filters out all but the neutrinos and some muons. Monte Carlo predictions of the relative beam composition and average energies of various neutrino types present in the beam are summarised in Table 1[13]. Variations in the relative beam compositions were used to estimate the systematic uncertainty due to the beam description in the parameters determined in Section 5.

Table 1: Average energies and relative abundances of neutrino types in the CERN-SPS wide band beam

\begin{tabular}{|l|c|c|}
\hline & Average $\mathrm{E}_{\nu}(\mathrm{GeV})$ & $\begin{array}{c}\text { Rel. flux } \\
\text { abundance }\end{array}$ \\
\hline$\nu_{\mu}$ & 23.5 & 1.000 \\
$\bar{\nu}_{\mu}$ & 19.2 & 0.061 \\
$\nu_{e}$ & 37.1 & 0.0094 \\
$\bar{\nu}_{e}$ & 31.3 & 0.0024 \\
\hline
\end{tabular}

\section{EXPERIMENTAL DETAILS}

\subsection{Apparatus}

The NOMAD detector, designed to search for a neutrino oscillation signal in the CERN SPS wide band neutrino beam, is described in detail in Ref. [14]. A side view of the detector is shown in Fig. 1. It consists of a number of subdetectors, most of which are located inside a $0.4 T$ dipole magnet with a volume of $7.5 \times 3.5 \times 3.5 \mathrm{~m}^{3}$. The relevant features for the present study will be briefly mentioned.

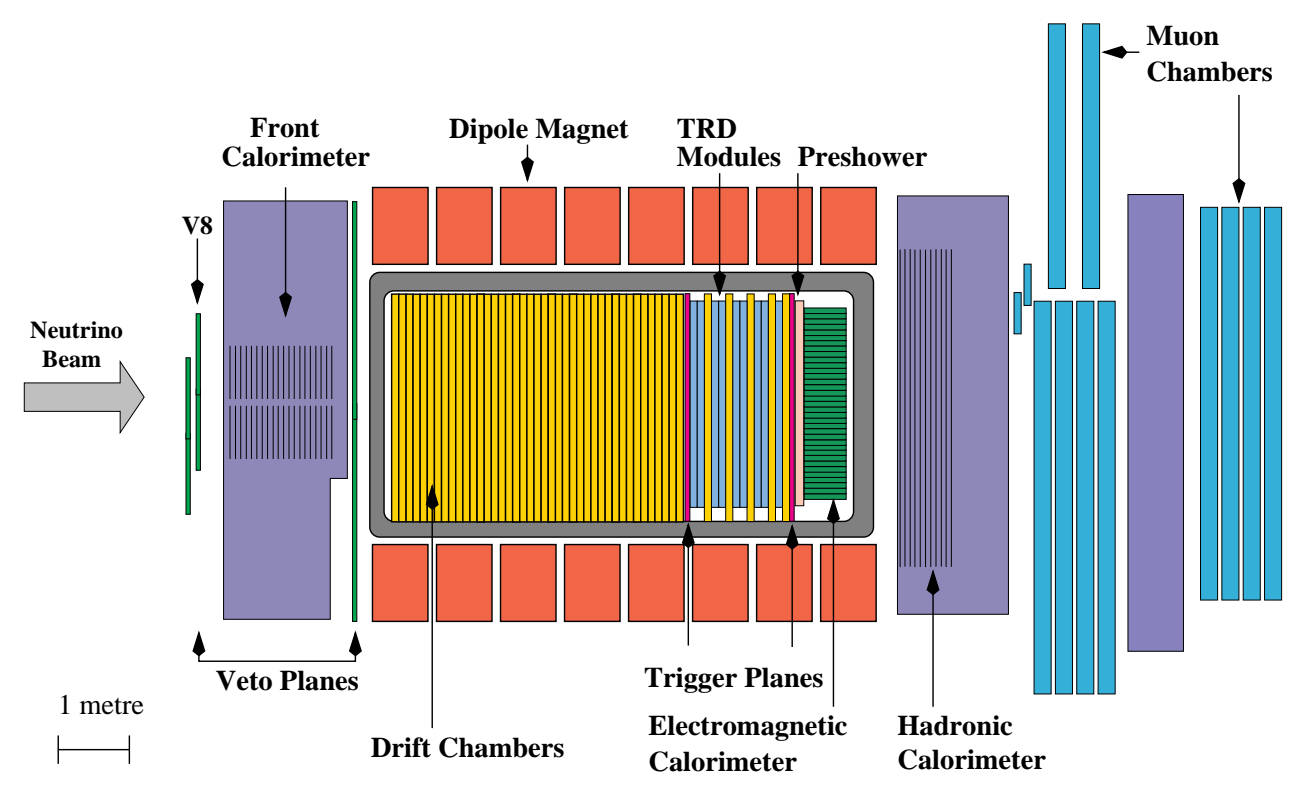

Figure 1: Side view of the NOMAD detector. The coordinate system has the $x$-axis into the plane of the figure, the $y$-axis directed up towards the top of the figure and the $z$-axis horizontal, approximately along the direction of the neutrino beam (which points upwards at an angle of $2.4^{\circ}$ with respect to the $z$-axis).

An iron-scintillator hadronic calorimeter, denoted the Front Calorimeter (FCAL), located upstream of the central part of the NOMAD detector, forms the target for the opposite sign dimuon study. It consists of 23 iron plates, $4.9 \mathrm{~cm}$ thick, separated by $1.8 \mathrm{~cm}$ air gaps. The first 20 gaps are instrumented with long scintillators which are read out at both ends by 3 in. photomultipliers. The dimensions of the scintillators are $175 \times 18.5 \times 0.6 \mathrm{~cm}^{3}$ and five consecutive scintillators are ganged together to form a module. Ten such modules are placed on top of one another to form a stack and there are four stacks aligned along the beam axis. The total instrumented region has a mass of $17.7 \mathrm{t}$ and is about 5 nuclear interaction lengths thick. The energy scale of the FCAL was calibrated in two stages. The relative calibration stage used high energy muons to derive the response of each FCAL module to the energy deposited by a minimum 
ionising particle (mip). The absolute energy scale, derived from a Monte Carlo study of neutral current neutrino interactions in which all the hadronic energy was contained within the FCAL volume, was then used to convert the energy deposition in units of mips to units of GeV. The energy calibration factor was determined to be $(2.95 \pm 0.02) \mathrm{mip} / \mathrm{GeV}$. Using this calibration factor the energy resolution of the FCAL is $\sigma / E=100 \% / \sqrt{E(G e V)}[15]$.

The tracking detector consists of 44 drift chambers grouped into 11 modules. This is followed by a transition radiation detector (TRD) to enhance the separation of electrons from pions. Five additional drift chambers are interleaved with the TRD modules. A preshower (PRS) detector precedes an electromagnetic calorimeter (ECAL), which consists of an array of lead glass Cerenkov counters. The energy resolution of the ECAL is $\sigma / E=1 \%+3.2 \% / \sqrt{E(G e V)}[14]$.

A hadronic calorimeter (HCAL) with an energy resolution of $\sigma / E=100 \% / \sqrt{E(G e V)}$ is installed behind the magnet coil and is followed by two muon detection stations consisting of large area drift chambers, the first after 8 and the second after 13 nuclear interaction lengths.

The FCAL trigger signal is obtained as the logical OR of the signals from each stack. A plane of scintillators, $V_{8}$, mounted on the upstream face of the FCAL as part of the veto, rejects charged particles either in the beam or from interactions in material upstream of NOMAD. Neutrino interactions in the FCAL are selected by a $\overline{V_{8}} \times F C A L$ trigger with a threshold set at $75 \mathrm{mV}$, corresponding to a deposited energy of approximately $2.0 \mathrm{GeV}$. The average rate of the $\overline{V_{8}} \times F C A L$ trigger is 6.5 per $10^{13}$ protons on target (p.o.t), with negligible cosmic ray background. The trigger livetime is $(89.5 \pm 2.2) \%[16]$.

\subsection{Monte Carlo simulations}

The opposite sign dimuon study reported here utilised two detector simulations. The first was a GEANT[17] based full detector simulation which included a detailed description of the detector geometry and which simulated the response of the detector to the particles produced in neutrino interactions. Neutrino events were simulated using a deep inelastic scattering event generator based on the LEPTO 6.1[18] and JETSET 7.4[19] packages. These events were then passed through the GEANT simulation and reconstructed in the same way as data. The full scale simulation was, however, too slow to use for the analysis presented in Section 5. To overcome this problem a fast detector simulation was constructed[15]. This simulation used detector response functions derived from the detailed simulation to model the detector acceptance, resolution and smearing, the efficiency of event selection and the details of the physics of both opposite sign dimuon and single muon charged current events. The parton densities used were those measured by the CCFR experiment[2]. These parton densities were found to reproduce well the shape of the kinematic distributions of the single muon NOMAD data[15]. Non-isoscalarity of the target and the violation of the Callan-Gross relation were included.

The fragmentation of the charm quark into a charmed hadron was modelled using the Collins-Spiller fragmentation function[20], which has been shown to describe adequately charm fragmentation data from the $e^{+} e^{-}$experiment CLEO $[21,22]$. This function is parametrised by

$$
D(z)=N_{0}\left(\frac{1-z}{z}+\frac{\epsilon_{C}(2-z)}{1-z}\right)\left(1+z^{2}\right)\left(1-\frac{1}{z}-\frac{\epsilon_{C}}{1-z}\right)^{-2}
$$

where $z=p_{h} / p_{h}^{\max }$ is the ratio of the momentum of the charmed hadron to the maximum momentum that the kinematics will permit that charmed hadron to possess, $N_{0}$ is a normalisation factor that ensures that the integral of $D(z)$ is unity and $\epsilon_{C}$ is a free parameter which has been determined by CLEO [22] to be $(0.6 \pm 0.1)$ and by CCFR[23] to be $(0.8 \pm 0.2)$. This study uses a value of $\epsilon_{C}=0.64 \pm 0.09$, the weighted average of the CLEO and CCFR results.

During fragmentation the charmed hadron acquires a momentum component transverse to the original direction of the charm quark. The size of this transverse component, $p_{T}$, was parametrised as $d N / d p_{T}^{2} \propto e^{-\beta p_{T}^{2}}$ where $p_{T}$ is in units of $\mathrm{GeV} / \mathrm{c}$. The value of the parameter $\beta$ was taken to be $1.1 \pm 0.3(\mathrm{GeV} / \mathrm{c})^{-2}$, as measured by the LEBC collaboration[24].

To model the relative proportion of charmed hadron species, we used the most recent analysis of the E531[9] data as quoted in Ref. [2]. In that analysis, the production fractions of charmed hadrons were determined to be $(60 \pm 6) \% D^{0},(26 \pm 6) \% D^{+},(7 \pm 5) \% D_{s}^{+}$and $(7 \pm 4) \% \Lambda_{c}^{+}$over a neutrino energy range of $30 \mathrm{GeV}$ to $200 \mathrm{GeV}$. The neutrino energy spectrum at NOMAD extends below $30 \mathrm{GeV}$, so to guard against systematic error a second analysis was performed using different production fractions, obtained from an earlier E531 analysis[25], in the range $E_{\nu}<30 \mathrm{GeV}$. The effect on the final result was negligible.

The branching ratios of the different semileptonic decay modes of the $\mathrm{D}$ meson and the $\Lambda_{c}$ baryon are listed in Table 2[12]. The D meson was decayed using a spin-1 vector matrix element in the case of the $K^{*}$ decay mode and a spin-0 pseudoscalar matrix element for the other decays[26]. As in JETSET[19], the decay of the $\Lambda_{c}$ baryon was carried out using a V-A matrix element to distribute the decay products. 
Table 2: The branching ratio of $\mathrm{D}$ meson and $\Lambda_{c}$ baryon semileptonic decays relative to their respective total muon semileptonic branching fraction.

\begin{tabular}{|l|c|}
\hline Mode & Relative branching ratio \\
\hline$D \rightarrow \nu \mu \pi$ & 0.05 \\
$D \rightarrow \nu \mu K$ & 0.67 \\
$D \rightarrow \nu \mu K^{*}$ & 0.28 \\
\hline$\Lambda_{c} \rightarrow \Lambda \nu \mu$ & 0.49 \\
\hline
\end{tabular}

\subsection{Data sample and event selection}

The present analysis is based on data collected during the 1995 and 1996 NOMAD runs. The total exposure was $1.8 \times 10^{19}$ p.o.t. Candidate opposite sign dimuon events were selected by the following criteria:

- Two muons of opposite electric charge had to be identified.

- The approximate longitudinal vertex position, identified as the midplane of the first stack in which there was significant energy deposition, must have occurred in one of the three most upstream stacks. The hadronic showers of events occurring in the last (fourth) stack are generally not longitudinally contained within the calorimeter and as a result the reconstructed properties of these events differ markedly from those events originating in the first three stacks.

- The transverse position of the vertex, obtained from energy sharing between the two ends of each module, had to be within the bounds of $-70 \mathrm{~cm}<x<70 \mathrm{~cm}$ and $-70 \mathrm{~cm}<y<70 \mathrm{~cm}$. This ensured that the hadronic shower was laterally contained.

- The distance between the positions of the muons, when extrapolated to the midplane of the first stack hit, had to be less than $20 \mathrm{~cm}$.

- The difference between the fitted times of each muon track at the first hit in the drift chambers had to be less than $5 \mathrm{~ns}$.

The dimuon events were categorised as originating from an incident neutrino or antineutrino by assuming that the primary muon (the muon produced at the leptonic vertex) was the one with the largest transverse momentum with respect to the beam direction. Monte Carlo studies indicate that this procedure identifies the correct primary muon with an efficiency of $(95 \pm 1) \%$ in neutrino induced interactions and $(93 \pm 1) \%$ in antineutrino interactions.

Denoting the 3 -momentum and energy of the primary muon by $\overrightarrow{p_{1}}$ and $E_{1}$ respectively, the 3momentum and energy of the secondary muon by $\overrightarrow{p_{2}}$ and $E_{2}$, the energy measured by the FCAL (the visible hadronic energy) by $E_{v i s}^{\text {had }}$ and letting $\hat{\vec{a}}$ represent a unit vector parallel to the beam direction, we may define the following kinematic quantities :

$-E_{v i s}^{\nu}=E_{1}+E_{2}+E_{v i s}^{\text {had }}$, the visible neutrino energy;

$-Q_{v i s}^{2}=2 E_{v i s}^{\nu}\left(E_{1}-\overrightarrow{p_{1}} \cdot \hat{\vec{a}}\right)-m_{\mu}^{2}$, the visible negative four-momentum transfer squared;

$-\nu_{v i s}=E_{2}+E_{v i s}^{\text {had }}$, the visible energy transferred to the hadronic system.

$-x_{v i s}=Q_{v i s}^{2} / 2 M \nu_{v i s}$, the visible Bjorken $\mathrm{x}$;

$-y_{v i s}=\nu_{v i s} / E_{v i s}^{\nu}$, the visible Bjorken $\mathrm{y}$;

In addition to the topological cuts listed earlier, a set of kinematic cuts were also applied. Both muons were required to have an energy greater than $4.5 \mathrm{GeV}$, primarily to reduce the meson decay background. The visible hadronic energy, $E_{v i s}^{\text {had }}$, was required to be more than $5 \mathrm{GeV}$ to ensure good hadronic energy reconstruction. Finally, badly reconstructed events (e.g. events in which the energy of the primary muon was too high to measure accurately) and events in regions where the simulation was considered to be unreliable were rejected by the criteria $x_{v i s}<1$ and $Q_{v i s}^{2}>1 \mathrm{GeV}^{2}$.

From a Monte Carlo simulation using the above criteria, the dimuon selection efficiency was estimated to be $(14 \pm 2) \%$. The largest loss of efficiency was due to the requirement that the secondary muon be identified as a muon. The average momentum of the secondary muon is approximately $5 \mathrm{GeV} / \mathrm{c}$. Requiring that it be observed in the muon chambers imposes an implicit momentum cut of at least 3 $\mathrm{GeV} / \mathrm{c}$, due to the rangeout of the muons within the FCAL itself or in the rest of the detector. This requirement, coupled with the steep secondary muon momentum spectrum, leads to a significant loss of events.

Figure 2 shows an example dimuon event from the NOMAD data. 


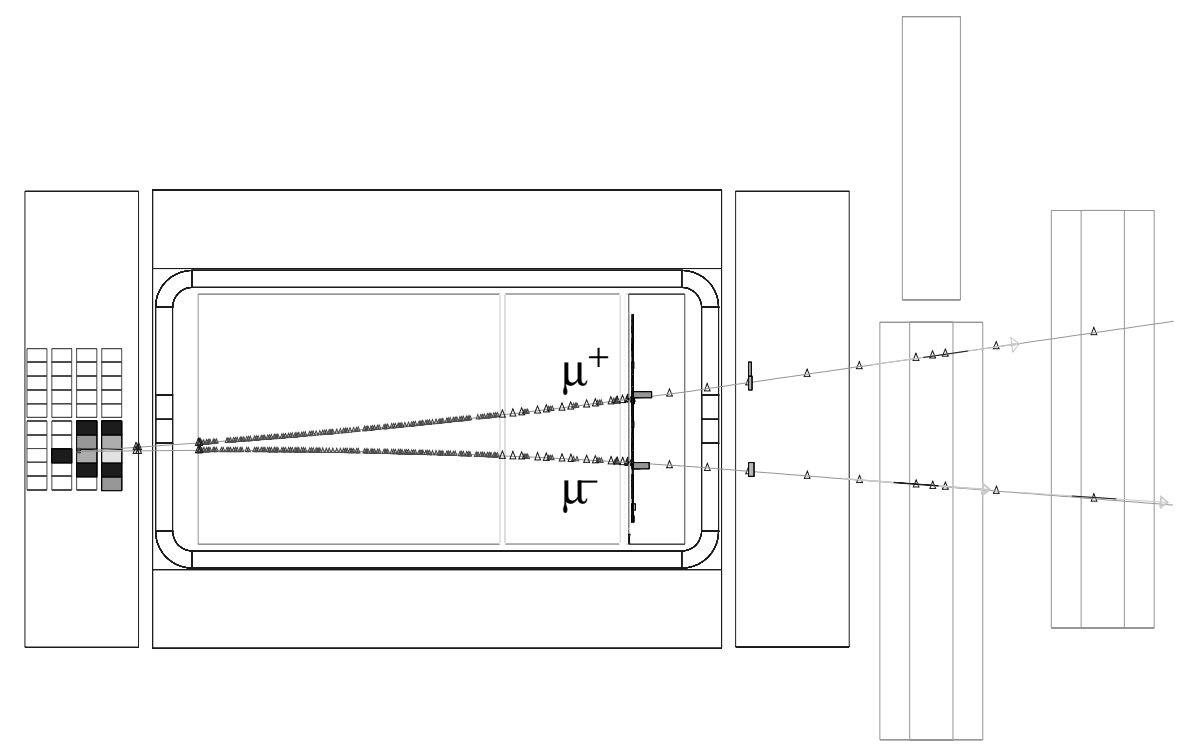

Figure 2: Example of an opposite sign dimuon event occurring at the end of Stack 2 in the FCAL. The two tracks are the oppositely charged muons in the event and the detector is shown from the side. The grey shading in each FCAL module indicates different levels of energy deposition.

\subsection{Background estimation}

The background to the opposite sign dimuon process arises from muonic decays of hadrons produced in the hadronic shower or by hadrons which punch through to the muon chambers, thereby simulating muons. The amount of background was estimated by studying dimuon events in which both muons had identical electric charge, the so-called like sign events. This class of events is dominated by the decay of hadrons (mostly pions and kaons) [27, 28] and hence, since the same physics processes give rise to the like sign events as to the opposite sign background events, can be used to estimate the background to the opposite sign dimuon signal.

The background subtraction algorithm, for any distribution A under study, was as follows :

- the number of like sign events in the data was determined.

- using the detailed Monte Carlo, the ratio, $\mathbf{R}$, of the number of reconstructed opposite sign background events to the number of reconstructed like sign events was calculated. Due to a combination of leading charge effects and slightly different acceptances for positive and negative muons, more opposite sign background events were observed than like sign events. This is illustrated in Table 3.

Table 3: The number of reconstructed opposite sign background events, like sign events and their ratio in simulated neutrino and antineutrino charged current event samples.

\begin{tabular}{|l|c|c|}
\hline & $\nu_{\mu}$ & $\bar{\nu}_{\mu}$ \\
\hline Initial number of events & 288,000 & 72,144 \\
Number of non-charm opposite & & \\
sign background events & $61 \pm 8$ & $10 \pm 3$ \\
Number of like sign events & $40 \pm 6$ & $5 \pm 2$ \\
\hline Background scale, R & $1.5 \pm 0.3$ & $2_{-1}^{+2}$ \\
\hline
\end{tabular}

- the same distribution for the like sign events in the data, $\mathbf{A}_{D A T A}^{L S D M}$, was scaled by $\mathbf{R}$.

- the scaled distribution was subtracted, bin-by-bin, from $\mathbf{A}$.

For this prescription to be valid it is important that the simulation describes both the shapes of the distributions of the like sign data and the production rate satisfactorily. The rate of observed like sign events normalised to the number of observed neutrino charged current events was measured to be $(2.7 \pm$ $0.1) \times 10^{-4}$ in the data, to be compared to $(2.7 \pm 0.4) \times 10^{-4}$ in the detailed simulation. ure 3 shows a comparison of the shapes of various distributions of like sign events in the data and the simulation. The agreement is good. 

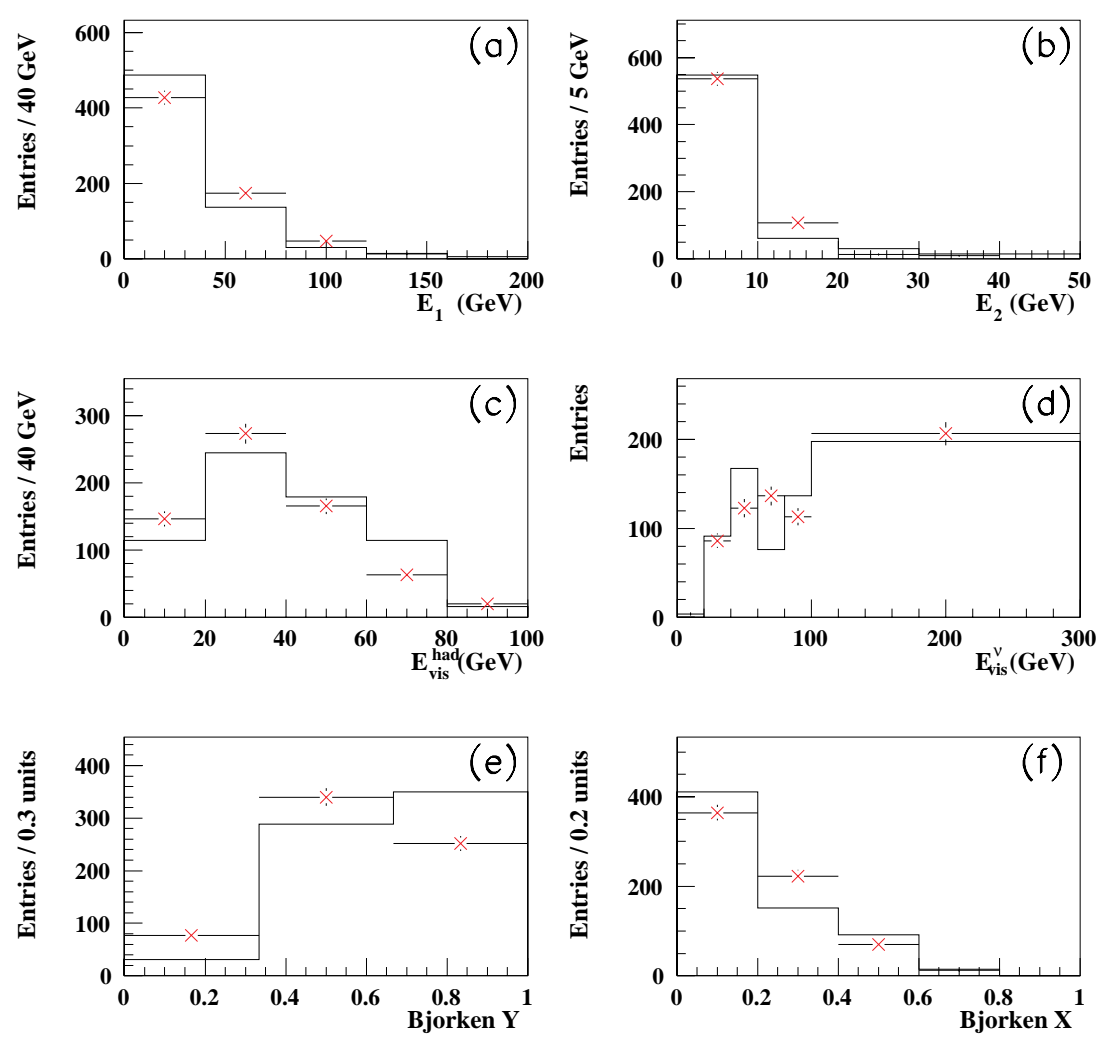

Figure 3: Comparison of the shapes of the kinematic distributions of like sign dimuon simulation (histogram) and data (points). Shown are the distributions of (a) the energy of the primary muon, (b) the energy of the secondary muon candidate, (c) the visible hadronic energy, (d) the visible neutrino energy, (e) Bjorken y and (f) Bjorken x. The distributions from the data and simulation have been normalised to equal area.

Other background sources such as trident production[29, 30], the overlap of a neutrino and an antineutrino event, or the production and subsequent muonic decay of a $J / \psi$ were found to be negligible in this analysis.

A total of 3590 leading $\mu^{-}$and 259 leading $\mu^{+}$events survived the selection process. Subtracting the background and correcting for contamination of the neutrino sample by antineutrino-induced dimuon events (and vice versa) yields an estimated number of 2714 neutrino-induced and 115 antineutrino-induced dimuon events. Table 4 summarizes the number of dimuon events in the data sample.

Table 4: The number of events with a leading $\mu^{-}$or $\mu^{+}$. Shown are the total number of opposite sign events observed, the total number of like sign events, the background estimate and the number of opposite sign events from charm production corrected for background and contamination of the neutrino(antineutrino) sample by the antineutrino(neutrino) sample. The background is estimated by scaling the number of like sign events by $1.5 \pm 0.3$ for the neutrino induced dimuons and by $2.0_{-1.0}^{+2.0}$ for the antineutrino sample. The uncertainty on the number of corrected events combines the statistical error on the raw opposite sign and like sign dimuon event numbers with the uncertainty on the background scaling factor and the errors on the neutrino and antineutrino dimuon event recognition efficiencies.

\begin{tabular}{|l|c|c|}
\hline & Leading $\mu^{-}$ & Leading $\mu^{+}$ \\
\hline Number of opposite sign & 3590 & 259 \\
events in data & 669 & 8 \\
$\begin{array}{l}\text { Like sign events in data } \\
\begin{array}{l}\text { Estimated number of opposite } \\
\text { sign background events }\end{array}\end{array}$ & 1004 & 16 \\
\hline $\begin{array}{l}\text { Number of opposite sign dimuon } \\
\text { events after background }\end{array}$ & & \\
$\begin{array}{l}\text { subtraction and correction } \\
\text { for cross-contamination }\end{array}$ & $2714 \pm 227$ & $115_{-41}^{+38}$ \\
\hline
\end{tabular}




\section{ANALYSIS}

\subsection{Description and results}

The production of opposite sign dimuon events is governed by four parameters : the charm quark mass, $m_{c}$, the proportion of strange to nonstrange quarks in the nucleon sea, usually determined by the parameter $\kappa=2 \bar{S} /(\bar{U}+\bar{D})$ (where $\bar{S}=\int_{0}^{1} x \bar{s}(x) d x, \bar{U}=\int_{0}^{1} x \bar{u}(x) d x$ and $\left.\bar{D}=\int_{0}^{1} x \bar{d}(x) d x\right)$, the CKM matrix element, $V_{c d^{1)}}$, and $B_{c}$, the average semileptonic branching ratio of charmed hadrons. Determinations of these parameters were made by fitting the observed kinematic distributions to model distributions obtained from a Monte Carlo simulation. To obtain the model predictions for different sets of production parameters, each simulated event in a "seed" event sample was given a weight proportional to the ratio of the differential cross sections for that event, calculated using the parameters for the new and seed samples. The seed sample consisted of approximately 140,000 neutrino dimuon events (after event selection) generated with $m_{c}=0.1 \mathrm{GeV} / \mathrm{c}^{2}, \kappa=0.36, V_{c s}=0.974[12]$ and $V_{c d}=0.221[12]$ using the fast Monte Carlo code described in Section 4.2. The charm quark mass at generation was chosen to be small to ensure that the fit was not biased by any mass thresholds in the seed sample.

Since the antineutrino sample was only a small fraction of the total event sample, it was expected that any determination of $V_{c d}$ would be less precise than the current value of $(0.221 \pm 0.003)$ and so $V_{c d}$ and $V_{c s}$ were fixed to their best known values.

A simultaneous fit for $m_{c}$ and $\kappa$ was performed using MINUIT[31] in order to check the degree of correlation between the two variables. The correlation was found to be small, allowing each parameter to be determined independently of the other. The mass of the charm quark was estimated by analysing the shape of the $E_{v i s}^{\nu}$ distribution after selection cuts had been applied, with $\kappa$ held constant. Once $m_{c}$ was determined, $\kappa$ was measured using the shape of the $x_{v i s}$ distribution. Finally, the average semileptonic branching ratio, $B_{c}$, was extracted by studying the observed rate of dimuons with respect to single muon events. The optimum value of each parameter was determined by a $\chi^{2}$ minimization procedure. The $E_{v i s}^{\nu}$ distribution was split into 6 bins ( 0 - $20-30-50-80-100-300 \mathrm{GeV}$ ) and the $x_{v i s}$ distribution was split into 5 bins $(0-0.05-0.1-0.3-0.5-1.0)$. The bins were defined so that the statistical uncertainty in each bin was approximately equal. Table 5 shows the fit results along with the statistical and systematic uncertainties on each parameter and the $\chi^{2}$ for each fit. The systematic uncertainties are discussed below. A comparison of distributions from a Monte Carlo sample generated with the best fit values of $m_{c}$ and $\kappa$ and data is shown in Fig. 4. The good agreement shows that the slow-rescaling model of charm quark production by neutrinos describes the data well.

Table 5: Fit results, statistical and systematic uncertainties and the fit $\chi^{2} /$ d.o.f (degree of freedom) for parameters of the dimuon cross section.

\begin{tabular}{|c|c|c|c|c|}
\hline Parameter & Result & $\begin{array}{c}\text { Statistical } \\
\text { Uncertainty }\end{array}$ & $\begin{array}{c}\text { Systematic } \\
\text { Uncertainty }\end{array}$ & $\begin{array}{c}\text { Fit } \chi^{2} \\
\text { per d.o.f }\end{array}$ \\
\hline$m_{c}$ & 1.3 & ${ }_{-0.3}^{+0.3}$ & ${ }_{-0.3}^{+0.3}$ & $6.1 / 5$ \\
$\kappa$ & 0.48 & ${ }_{-0.07}^{+0.09}$ & ${ }_{-0.12}^{+0.17}$ & $3.3 / 4$ \\
$B_{c}$ & 0.095 & ${ }_{-0.007}^{+0.007}$ & ${ }_{-0.014}^{+0.014}$ & - \\
\hline
\end{tabular}

\subsection{Systematic errors}

Systematic errors on the fit results arise from uncertainties in the theoretical model, uncertainties in the exact value of reconstructed kinematic variables which define the analysis cuts and uncertainties in the background subtraction procedure. The theoretical uncertainties were studied by varying the parameters of the model by their quoted errors, repeating the fit, and observing the shift in the best fit values of $m_{c}, \kappa$ and $B_{c}$. The effect of the finite resolution on the cut variables was studied by varying each cut around its central value and repeating the fit. The systematic uncertainty due to the background scale was estimated by varying the scale within its errors (see Table 3 ). The parton densities used in this analysis were based on the Buras-Gaemers model with nine free parameters[2, 32]. The impact of the uncertainties in these distribution functions on the charm parameters was studied by varying each of the nine parameters by their quoted error and repeating the fit. The uncertainty due to the beam description was estimated by weighting the seed sample events by the ratio of the flux predictions from two different beam descriptions and repeating the analysis. One beam description uses a version of FLUKA which is incorporated into the GEANT[17] package and was the default simulation for this analysis. The other beam simulation (the so called empirical parametrisation) uses cross sections for meson and hadron production in $\nu$-Be

\footnotetext{
1) The CKM element, $V_{c s}$, can be obtained from the unitarity condition of the CKM matrix.
} 

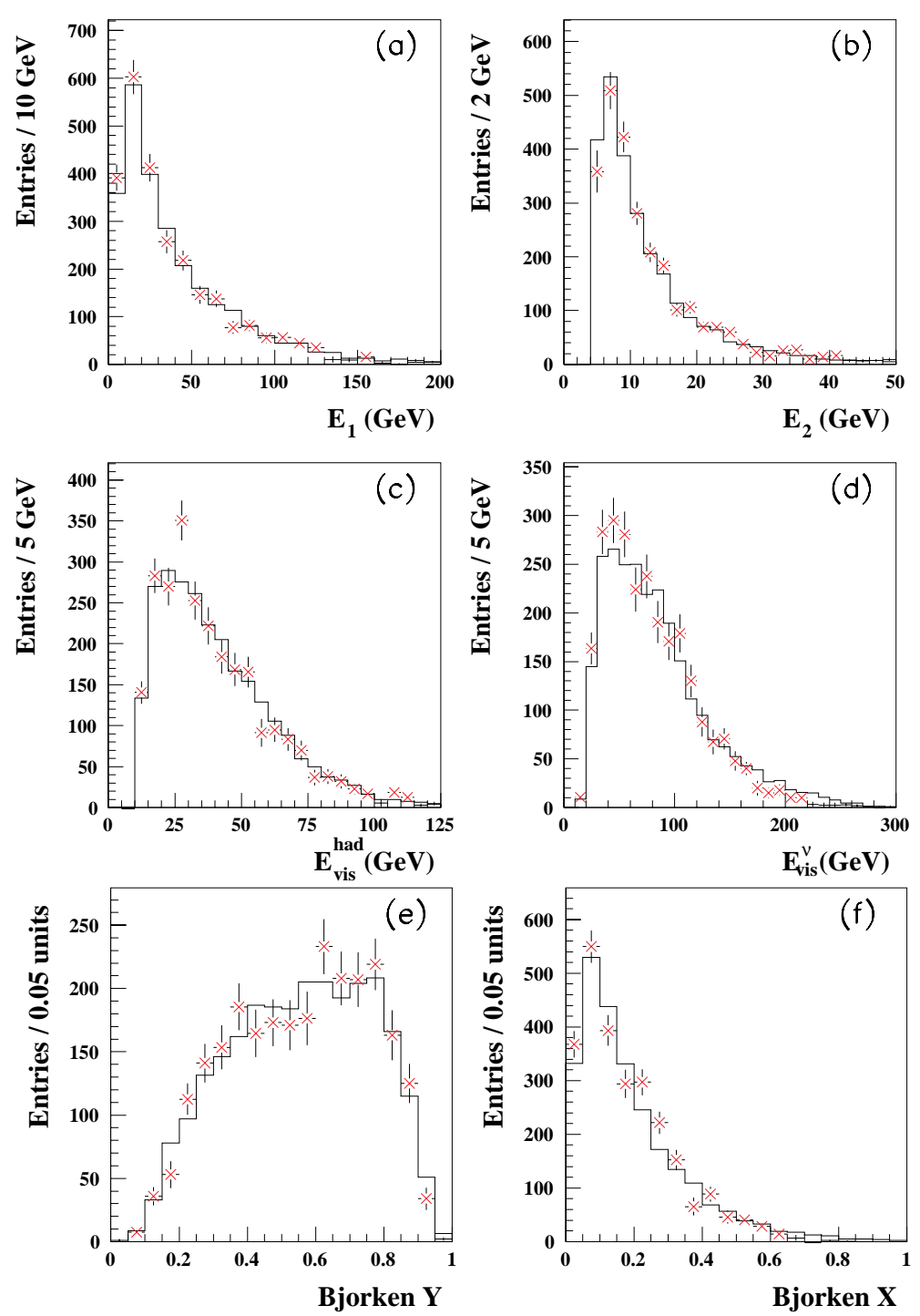

Figure 4: Comparison of the shapes of the kinematic distributions of an opposite sign dimuon simulation (histogram) and background subtracted data (points). Shown are distributions of (a) the energy of the primary muon, (b) the energy of the secondary muon, (c) the visible hadronic energy, (d) the visible neutrino energy, (e) visible Bjorken y and (f) visible Bjorken $\mathrm{x}$. The distributions from the data and simulation have been normalised to equal area.

interactions which have been determined from the analysis of $\nu_{\mu}, \bar{\nu}_{\mu}, \nu_{e}$ and $\bar{\nu}_{e}$ energy spectra observed in the NOMAD data and which agree with empirically measured production yields[33].

A summary of the systematic errors is presented in Table 6 . The effect of varying the value of the cut on Bjorken $x$ was negligible in all cases.

\section{DISCUSSION}

A comparison of these results with those reported by the CDHS[1], CHARM II[4], CCFR[3] and FMMF[5] experiments is shown in Table 7 . All parameters measured in this study are compatible with previous experiments.

Within the framework of leading order QCD, an SU(3) symmetric sea would yield a value of $\kappa=1$. The smaller value observed supports the conclusion that the size of the strange sea is suppressed with respect to the size of the non-strange sea. The strange sea content of the nucleon may be defined by $\eta_{s}=2 S /(U+D)$. Using the measured value of $\kappa$ and taking the total antiquark to quark ratio, $\bar{Q} / Q$, to be 0.177 from the CCFR[3] parton density parametrisation yields

$$
\eta_{s}=0.071_{-0.009}^{+0.011+0.020}+015
$$

which is consistent with previous publications $[3,4]$. The average branching ratio determined in this study is compatible with the values obtained by other experiments (see Table 7). 
Table 6: Contributions to the total systematic uncertainties in the fitted parameters.

\begin{tabular}{|c|c|c|c|}
\hline \multicolumn{4}{|c|}{ Theoretical Uncertainties } \\
\hline Source of Uncertainty & $\Delta m_{c}$ & $\Delta \kappa$ & $\Delta B_{c}$ \\
\hline Background Scale & $\begin{array}{l}+0.16 \\
-0.17\end{array}$ & $\begin{array}{l}+0.10 \\
+0.06\end{array}$ & $\begin{array}{l}+0.0070 \\
-0.0080\end{array}$ \\
\hline Fragmentation parameter & +0.03 & +0.005 & +0.0011 \\
\hline$p_{T}$ distribution $(\beta)$ & $\begin{array}{l}-0.04 \\
+0.03 \\
-0.02\end{array}$ & $\begin{array}{l}-0.003 \\
+0.004 \\
-0.007\end{array}$ & $\begin{array}{l}-0.0023 \\
+0.0013\end{array}$ \\
\hline$m_{c}$ & - & $\begin{array}{l}+0.07 \\
-0.05\end{array}$ & $\begin{array}{l}-0.002 \\
+0.002 \\
-0.003\end{array}$ \\
\hline$\kappa$ & $\begin{array}{l}+0.07 \\
-0.03\end{array}$ & - & +0.012 \\
\hline$V_{c d}$ & $\begin{array}{l}-0.03 \\
+0.01 \\
-0.01\end{array}$ & $\begin{array}{l}+0.05 \\
+0.02\end{array}$ & $\begin{array}{l}-0.0050 \\
+0.0001 \\
-0.0001\end{array}$ \\
\hline$V_{c s}$ & $\begin{array}{l}+0.01 \\
-0.01\end{array}$ & $\begin{array}{l}+0.02 \\
-0.01\end{array}$ & $\begin{array}{l}+0.0001 \\
-0.0001\end{array}$ \\
\hline Structure Functions & \pm 0.06 & \pm 0.02 & \pm 0.001 \\
\hline Target mass correction & \pm 0.11 & \pm 0.04 & \pm 0.002 \\
\hline Radiative correction & \pm 0.05 & \pm 0.05 & \pm 0.001 \\
\hline \multicolumn{4}{|c|}{ Experimental Uncertainties } \\
\hline Beam description & \pm 0.01 & \pm 0.01 & \pm 0.0001 \\
\hline Fiducial Volume & $\begin{array}{l}+0.02 \\
-0.03\end{array}$ & $\begin{array}{l}+0.01 \\
-0.01\end{array}$ & +0.0002 \\
\hline Difference between fitted & & & \\
\hline time of muons at first hit & +0.01 & +0.003 & +0.0001 \\
\hline $\begin{array}{l}\text { in drift chambers } \\
\text { Distance between muons at }\end{array}$ & -0.01 & -0.001 & -0.0001 \\
\hline interaction point & +0.05 & +0.02 & +0.0011 \\
\hline$Q_{v i}^{2}$ & $\begin{array}{l}-0.07 \\
+0.01 \\
+0.02\end{array}$ & $\begin{array}{l}-0.02 \\
+0.01\end{array}$ & $\begin{array}{l}-0.0020 \\
+0.0003\end{array}$ \\
\hline$E_{\text {vis }}^{\text {had }} \mathrm{cut}$ & $\begin{array}{l}-0.03 \\
+0.09\end{array}$ & $\begin{array}{l}-0.03 \\
+0.001\end{array}$ & $\begin{array}{l}-0.0008 \\
+0.0003\end{array}$ \\
\hline Cut on energy of & & -0.004 & -0.0002 \\
\hline primary muon & $\begin{array}{l}+0.04 \\
-0.03\end{array}$ & ${ }_{-0.006}^{+0.006}$ & $\begin{array}{l}+0.0002 \\
-0.0001\end{array}$ \\
\hline Cut on energy of & & & \\
\hline secondary muon & $\begin{array}{l}+0.05 \\
{ }_{-0.03}\end{array}$ & $\begin{array}{l}+0.004 \\
{ }_{-0.003}\end{array}$ & $\begin{array}{l}+0.0009 \\
-0.0008\end{array}$ \\
\hline Background shape & $\begin{array}{l}+0.06 \\
{ }_{-0.06}\end{array}$ & $\begin{array}{l}+0.02 \\
-0.03\end{array}$ & - \\
\hline Hadronic energy scale & $\begin{array}{l}+0.07 \\
-0.04\end{array}$ & $\begin{array}{l}+0.08 \\
+0.04\end{array}$ & $\begin{array}{l}+0.0003 \\
-0.0006\end{array}$ \\
\hline Total systematic uncertainty & $\begin{array}{l}+0.27 \\
-0.26\end{array}$ & $\begin{array}{l}+0.17 \\
-0.12\end{array}$ & $\begin{array}{l}+0.014 \\
-0.013\end{array}$ \\
\hline
\end{tabular}

Table 7: Comparison of CDHS, NOMAD, CHARM II, CCFR and FMMF Leading Order results. The experiments are listed from top to bottom in order of increasing average neutrino energy. Also shown are the numbers of neutrino- and antineutrino-induced opposite sign dimuon events observed by each experiment.

\begin{tabular}{|c|c|c|c|c|c|c|}
\hline Experiment & $<E_{\nu_{\mu}}>$ & $N_{2 \mu}$ for $\nu$ & $N_{2 \mu}$ for $\bar{\nu}$ & $m_{c}\left(\mathrm{GeV} / \mathrm{c}^{2}\right)$ & $\kappa$ & $B_{c}$ \\
\hline CDHS & 20.0 & 9922 & 2123 & & $0.47_{-0.08-0.05}^{+0.08+0,05}$ & $0.084_{-0.014}^{+0.014}$ \\
\hline NOMAD & 23.6 & 2714 & 115 & $1.3_{-0.3-0.3}^{+0.3+0.3}$ & $0.48_{-0.07-0.12}^{+0.09+0.17}$ & $0.095_{-0.007}^{+0.007}{ }_{-0.013}^{+0.014}$ \\
\hline CHARM II & 23.6 & 3100 & 700 & $1.8_{-0.3-0.3}^{+0.3}$ & $0.39_{-0.06-0.07}^{+0.07}+0.07$ & $0.091_{-0.007}^{+0.007}+0.007$ \\
\hline $\begin{array}{l}\text { CCFR } \\
\text { FMMF }\end{array}$ & 140.0 & $\begin{array}{c}4503 \\
393\end{array}$ & 632 & $1.3_{-0.2}^{+0.2+0.1}$ & $\begin{array}{r}0.44_{-0.09+0.07}^{+0.09}+0.02 \\
0.41^{+0.08+0.103}\end{array}$ & $0.109_{-0.008}^{+0.008+0.006}$ \\
\hline
\end{tabular}

The slow rescaling model may be best tested by investigating the rate of charm-induced dimuon production relative to single muon production as a function of neutrino energy. This relative rate is shown in Fig. 5. Data have been corrected bin-by-bin, using Monte Carlo simulation, for the effects of geometric acceptance, reconstruction efficiency, energy loss due to the charm decay neutrino and the energy resolution. The energy resolution correction takes into account the correlation between data points. The hadron energy scale is established by the measured single muons in the FCAL; the muon energy via the measured magnetic field in NOMAD. The statistical and systematic errors have been added in quadrature. The NOMAD data are compatible with the CCFR[3] and the CDHS[1] data, and extend to the crucial low neutrino energy region. The results clearly exhibit an energy dependence characteristic of heavy quark production and are consistent with the slow-rescaling hypothesis. The measured charm excitation curve is the most precise to date in the energy region below $50 \mathrm{GeV}$. 


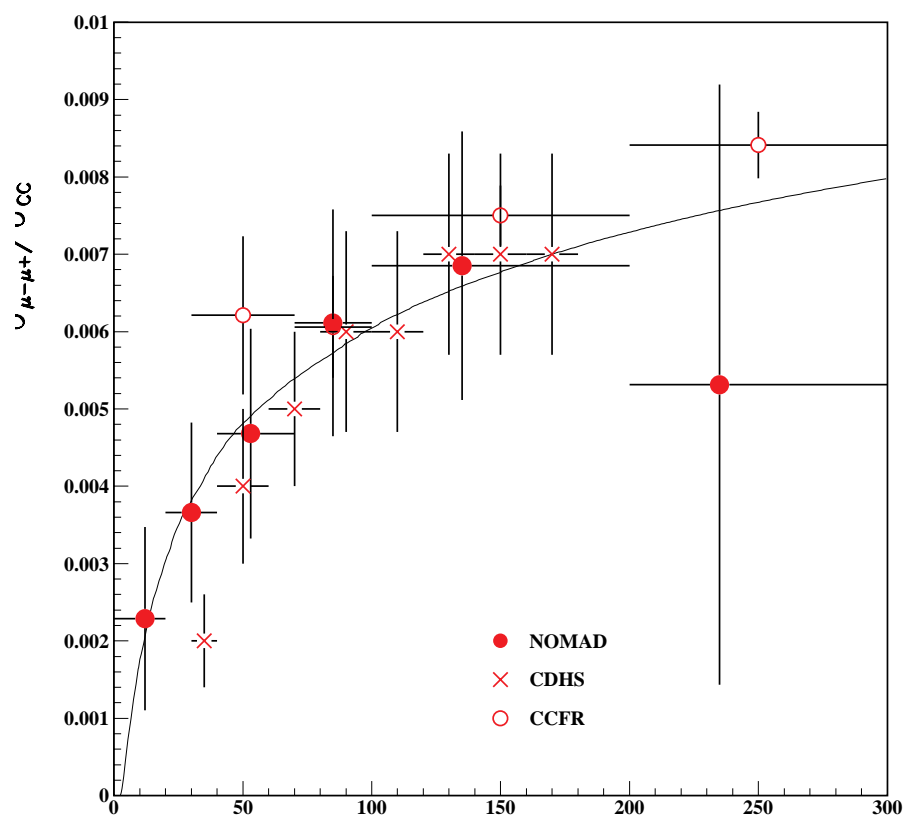

Figure 5: Neutrino-induced opposite sign dimuon rates corrected for acceptance and kinematic effects assuming a charm mass of $1.3\left(\mathrm{GeV} / \mathrm{c}^{2}\right)$. The NOMAD results are displayed as closed circles. Also shown are the CCFR (open circles) and the CDHS results (crosses). The curve is the theoretical ratio of cross sections assuming the slow rescaling mechanism in Leading Order QCD, a charm mass of $1.3\left(\mathrm{GeV} / \mathrm{c}^{2}\right)$, a value for $\kappa$ of 0.48 , an average semileptonic branching ratio of 0.095 and the validity of the CCFR structure functions.

\section{SUMMARY}

Dimuon production by neutrinos in the NOMAD detector has been studied in the context of Leading Order QCD. The data are consistent with the hypothesis of charm production via the slow rescaling model. Within this framework, the charm quark mass, $m_{c}=1.3_{-0.3}^{+0.3}{ }_{-0.3}^{+0.3}\left(\mathrm{GeV} / \mathrm{c}^{2}\right)$, and the strange content of the nucleon, $\eta_{s}=0.071_{-0.009}^{+0.011}{ }_{-0.015}^{+0.020}$, have been determined and shown to be compatible with the results of previous leading order QCD analyses. The strange quark content has been shown to be suppressed relative to the non-strange sea quarks by a factor of $\kappa=0.48_{-0.07-0.12}^{+0.09+0.17}$. The average semileptonic branching ratio, determined to be $B_{c}=0.095_{-0.007}^{+0.007}{ }_{-0.013}^{0.014}$, is consistent with the interpretation that it is a weakly rising function of neutrino energy, arising from different energy dependencies of the various charmed hadron production rates.

\section{ACKNOWLEDGEMENTS}

We thank the management and staff of CERN and of all participating institutes for their support. Particular thanks are due to the CERN accelerator and beam-line staff for the magnificent performance of the neutrino beam. The following funding agencies have contributed to this experiment: Australian Research Council (ARC) and Department of Industry, Science, and Resources (DISR), Australia; Institut National de Physique Nucléaire et Physique des Particules (IN2P3), Commissariat à l'Energie Atomique (CEA), Ministère de l'Education Nationale, de l'Enseignement Supérieur et de la Recherche, France; Bundesministerium für Bildung und Forschung (BMBF, contract 05 6DO52), Germany; Istituto Nazionale di Fisica Nucleare (INFN), Italy; Russian Foundation for Basic Research, Institute for Nuclear Research of the Russian Academy of Sciences, Russia; Fonds National Suisse de la Recherche Scientifique, Switzerland; Department of Energy, National Science Foundation (grant PHY-9526278), the Sloan and the Cottrell Foundations, USA. F.J.P. Soler is supported by a TMR Fellowship from the European Commission. We thank A. Ferrari and P.R. Sala for their contributions toward our beam simulation.

We also thank our secretarial staff, Jane Barney, Marie-Anne Huber, Jennifer Morton, Rachel Phillips, and Mabel Richtering, and the following people who have worked with the collaboration on the preparation and the data collection stages of NOMAD: M. Anfreville, M. Authier, A. Beer, V. Bonaiti, A. Castera, O. Cloué, C. Détraz, L. Dumps, C. Engster, G. Gallay, W. Huta, E. Lessmann, J. Mulon, J.P. Passérieux, P. Petitpas, J. Poinsignon, M. Richtering, M. Serrano, C. Sobczynski, S. Soulié, L. Visentin, P. Wicht. 


\section{References}

[1] H. Abramowicz et al., Z. Phys. C15, 19-31 (1982)

[2] A.O.Bazarko, Ph.D. thesis, Columbia University, 1994.

[3] S. A. Rabinowitz et al., CCFR Collaboration, Phys. Rev. Lett. 70, 134-137 (1993); C. Foudas et al., CCFR Collaboration, Phys. Rev. Lett. 64, 1207-1210 (1990); A. O. Bazarko et al., CCFR Collaboration, Z.Phys. C65, 189-198 (1995)

[4] P. Vilain et al., Eur. Phys. J. C11, 19 (1999)

[5] B. Strongin et al., Phys. Rev. D43, 2778-2786 (1991)

[6] N.J.Baker et al., Phys. Rev. D43, 2765 (1991)

[7] G. Gerbier et al., Z. Phys. C29, 15 (1985)

[8] V. Jain et al., Phys. Rev. D41, 2057-2073 (1990)

[9] N. Ushida et al., Phys. Lett. B121, 292-296 (1983)

[10] R. G. Ammar et al., Z. Phys. C45, 557-567 (1990)

[11] H. Georgi and H. D. Politzer, Phys. Rev. D14, 1829 (1976)

[12] Review of Particle Physics, Particle Data Group, Euro. Phys. J. C3, (1998)

[13] G. Collazuol et al., CERN-OPEN-98-032 (1998)

[14] J. Altegoer et al., Nucl. Inst. and Meth. A404, 96 (1998)

[15] S. Boyd, Ph.D. thesis, University of Sydney, 1998.

[16] J. Altegoer et al., Nucl. Inst. and Meth. A428, 299-316 (1999)

[17] The NOMAD simulation program is based on GEANT 3.21, CERN Program Library Long Writeup W5013.

[18] G. Ingelman, in Proc. Physics at HERA, 1992, edited by W. Buchmueller and G. Ingelman (DESY, Hamburg, 1992), p. 1366

[19] T. Sjöstrand, Comp. Phys. Comm. 82, 74-89 (1994); T. Sjöstrand, CERN-TH/93-7112 (1993)

[20] P. Collins and T. Spiller, J. Phys. G11, 1289-1298 (1985)

[21] D. Andrews et al, Nucl. Inst. and Meth. A211, 47-71 (1983)

[22] D. Bortoletto et al., Phys. Rev. D37, 1719-1743 (1988)

[23] A.O.Bazarko et al., Z. Phys. C65, 189-198 (1995)

[24] M. Aguilar-Benitez et. al., Phys. Lett. B123, 98-102 (1983)

[25] N. Ushida et al., Phys. Lett. B206, 375-379 (1988)

[26] J.D. Richman and P.R.Burchat Rev. Mod. Phys., Vol\# 67, No. 4. 893-946 (1995)

[27] K. Lang et al., Z. Phys. C33, 483 (1987)

[28] C. Baltay et al., Phys. Rev. Lett. 55, 2543-2546 (1985)

[29] D. Geiregat et al, Phys. Lett. B245, 271 (1990)

[30] S.R. Mishra et al, Phys. Rev. Lett. 66, 3117-3120 (1991)

[31] F. James, MINUIT 94.1, CERN Program Library Long Writeup D506.

[32] A.J. Buras and K.J.F. Gaemers, Nucl. Phys. B132, 249 (1978)

[33] Atherton et al., CERN 80-07, August 1980. G. Ambrosini et al., Phys. Lett. B420, 225-232 (1998); G. Ambrosini et al., Phys. Lett. B425, 208-214 (1998); G. Ambrosini et al., Eur. Phys. J. C10, 605 (1999) 